\title{
Endoplasmic reticulum stress-induced PCD and caspase-like activities involved
}

\section{Yao-Min Cai, Jia Yu and Patrick Gallois*}

Faculty of Life Sciences, University of Manchester, Manchester, UK

\section{Edited by:}

Patrick Schäfer, University of Warwick, UK

\section{Reviewed by:}

Nuria Sanchez Coll, Centre for Research in Agricultural Genomics, Spain

Christine Gietl, Technische

Universitaet Muenchen, Germany

\section{${ }^{*}$ Correspondence:}

Patrick Gallois, Faculty of Life Sciences, The Michael Smith Building, University of Manchester, Oxford Road, Manchester, M13 9PT, UK e-mail:patrick.gallois@ manchester.ac.uk
Plant cells, like cells from other kingdoms, have the ability to self-destruct in a genetically controlled manner. This process is defined as Programmed cell death (PCD). PCD can be triggered by various stimuli in plants including by endoplasmic reticulum (ER) stress. Research in the past two decades discovered that disruption of protein homeostasis in the ER could cause ER stress, which when prolonged/unresolved leads cells into PCD. ER stress-induced PCD is part of several plant processes, for instance, drought and heat stress have been found to elicit ER stress-induced PCD. Despite the importance of ER stress-induced PCD in plants, its regulation remains largely unknown, when compared with its counterpart in animal cells. In mammalian cells, several pro-apoptotic proteases called caspases were found to play a crucial role in ER stress-induced PCD. Over the past decade, several key proteases with caspase-like enzymatic activity have been discovered in plants and implicated in PCD regulation. This review covers what is known about caspase-like enzymatic activities during plant ER stress-induced PCD and discusses possible regulation pathways leading to the activation of relevant proteases in plants.

\section{Keywords: programmed cell death, VPE, proteasome, UPR, plant caspases}

\section{INTRODUCTION}

Plant cells have the ability to self-destruct in a controlled manner. This process is called programmed cell death (PCD). Unlike death as a consequence of physical damage, which is not controlled by the cell itself, PCD is genetically regulated. PCD is a versatile tool for plants to cope with various situations or needs. In development, tracheary elements for example, undergo PCD to form the xylem (Groover et al., 1997; Bollhöner et al., 2012). PCD also is used in plant defense. When plant leaves are attacked by pathogens, PCD can be elicited locally where the tissue is invaded. In this case, PCD contributes to reducing the growth of the pathogen and possibly its spreading into other parts of the plant (Greenberg, 1996). Among the various stresses that induce plant PCD, endoplasmic reticulum (ER) stress is a new comer and is gaining more and more attention. The ER is an essential organelle. One of its functions is folding and $\mathrm{N}$-glycosylation of secreted proteins. If for various reasons these processes do not function properly, misfolded proteins will accumulate inside the ER and cause ER stress. Cells experiencing ER stress will try to restore ER homeostasis by inducing the expression of unfolded-protein-response (UPR) genes. The products of these genes help cells escape ER stress by increasing the protein folding capacity and secretion ability within the ER. However, if the mounted rescue response is overwhelmed by ER stress, cells will eventually enter PCD. Such an ER stress-induced PCD has been observed in plants when Arabidopsis seedlings were treated with increasing concentration of PEG8000, which mimics drought. ER stress was induced and eventually resulted in PCD of root cells (Duan et al., 2010). In addition, when plants are challenged with biotic stress, for example a pathogen, ER stress is observed (Moreno et al., 2012) and this may contribute to the induction of PCD. In the past few years, there has been several reports on how ER stress regulators regulate UPR genes in plant cells (Liu and Howell, 2010; Howell, 2013). However, we still have a limited picture of the PCD pathway components activated when ER stress is unresolved. Among PCD regulators, caspases are central components that mediate animal PCD pathways in response to various stimuli, including ER stress. Caspases are cysteine-dependent aspartate specific proteases. As the name suggests, caspases cleave their substrates after an aspartate residue (in P1 position in the cleavage site). This led to the development of synthetic tetra-peptides conjugated with fluorescent reporters to determine caspase enzymatic activities. For example, $\operatorname{Tyr}(\mathrm{Y})-\mathrm{Val}(\mathrm{V})-\mathrm{Ala}(\mathrm{A})-\mathrm{Asp}(\mathrm{D})$ is used to measure caspase-1 activity because it is the four amino acid pattern preferentially cleaved by caspase- 1 . Of note, YVAD can also be cleaved in vitro by other caspases or unrelated proteases such as legumains (Rotari et al., 2001; McStay et al., 2007). In plants, enzymatic assays using these synthetic caspase substrates have directly or indirectly implicated proteases with caspaselike activities in ER stress-induced PCD (Zuppini et al., 2004; Costa et al., 2008). The term caspase-like is used because there is no caspase ortholog in plant genomes and the plant proteases closest to animal caspases, the metacaspases, are unable to cleave synthetic caspase substrates and have instead Arg specificity. The plant caspase activities must therefore originate from proteases unrelated to animal caspases. Using various experimental systems, several research groups were able to identify plant proteases that possessed caspase-like enzymatic activities and regulated PCD. Plant proteases related to caspases and proteases with caspase-like activities have been extensively reviewed (Van der Hoorn, 2008; Xu and Zhang, 2009; Tsiatsiani et al., 2011; 
Vartapetian et al., 2011). This review focuses on the caspase-like activities linked to ER-stress induced PCD and comments on the proteases involved.

\section{COMPARISON OF THE REGULATION OF ER STRESS-INDUCED PCD BETWEEN PLANT AND OTHER MODEL ORGANISMS}

As hinted above, PCD is an evolutionary conserved consequence of unresolved ER stress. PCD can be induced experimentally by a prolonged treatment using an ER stress-inducing chemical. Signal transduction from ER stress to apoptosis in mammalian cells has been established, although it is not fully complete. Three ER tethered proteins: protein kinase RNA-like ER kinase (PERK), activating transcription factor (ATF6) and inositolrequiring enzyme (IRE1 $\alpha$ ) are involved in the regulation of ER stress-induced apoptosis in mammalian cells. Inside the ER lumen, the protein chaperone binding immunoglobulin protein (BiP) contributes to both refolding of misfolded proteins and activation of PERK, ATF6, and IRE1 $\alpha$. During ER stress, PERK activation is due to its dissociation from BiP. Active PERK phosphorylates eukaryotic initiation factor 2 (eIF2) which results in translation inhibition (Harding et al., 1999; Harding et al., 2000). However, not all protein translation is blocked and a cAMP response element-binding transcription factor ATF4 escapes the inhibition of protein translation (Harding et al., 2003). Although many ATF4 target genes are pro-survival, one of ATF4 target genes, C/EBP homolog protein (CHOP), is pro-apoptotic (Zinszner et al., 1998). The mechanism of CHOP-induced apoptosis relies on (1) Restoration of translation via dephosphorylation of eIF2 by a protein phosphatase 1 (GADD34), which is a target of CHOP; (2) Inhibition of the pro-survival serine/threonine kinase Akt; (3) Repression of anti-apoptotic protein B-cell lymphoma 2 (BCL-2) expression level. In addition to PERK, ATF6 is involved in regulating CHOP expression as the CHOP gene is a direct target of ATF6 (Yoshida et al., 2000). However, not many evidences support that ATF6 is directly involved in the apoptosis signal pathway, apart from that ATF6 is able to increase CHOP transcript level. Finally, in the IRE1 $\alpha$ branch, activation of IRE1 $\alpha$ is also through dissociation from BIP (Szegezdi et al., 2006). Active IRE $1 \alpha$ is able to recruit TNF-receptor-associate factor 2 (TRAF2), which in turn recruit apoptosis-signal-regulating kinase (ASK1; Nishitoh et al., 1998). The IRE1 $\alpha$-TRAF2-ASK1 complex activates c-Jun N-terminal kinase (JNK). Activation of JNK activates downstream pro-apoptotic signals. For instance, JNK represses BCL-2 via phosphorylation (Davis, 2000). Downstream of PERK, ATF6, and IRE1 $\alpha$ mediating signals is the activation of caspases. Caspase-12 (in rodent) and caspase-4 (in human) were reported to mediate a caspase cascade in ER stress-induced PCD in mammalian cells (Nakagawa et al., 2000). A recent report implicated caspase- 2 in ER stress-induced PCD of mouse embryonic fibroblasts (MEFs). Under severe ER stress, IRE1 $\alpha$, via its $\alpha$ RNase activity, degrades a selective set of miRNAs which repressed caspase-2 expression level (Upton etal., 2012). Supporting the importance of IRE1 $\alpha$ in this process, no expression of caspase-2 and no activation of caspase-3 were observed in IRE1 $\alpha$ knock out MEFs, and knock out of the pro-apoptotic BAX/BAK did not affect caspase-2 expression level. The discovery that caspase-2 expression level was controlled by IRE1 $\alpha$ and not by BAX/BAK suggested a direct signal pathway from IRE1 $\alpha$ to caspase- 2 and then to the mitochondrion apoptotic pathway involving BAX/BAK (Upton et al., 2012).

When looking into the ER stress-induced PCD signal pathway in plants, many of the regulators discovered in mammalian ER stress-induced apoptosis are missing from the genome sequences or at least functional equivalent are yet to be reported. For example, a PERK homolog has not been identified in plants and Kamauchi et al. (2005) reported that, unlike in animal cells, ER stress had no effect on protein translation in Arabidopsis. For this the authors used $S^{35}$ labeled methionine and cysteine to measure nascent protein expression level under ER stress in Arabidopsis. In addition, the phosphorylation level of eIF2 $\alpha$ showed a decrease rather than the increase described in animal cells (Kamauchi et al., 2005). All these experiments suggested that ER stress does not induce translation attenuation in plant cells. So it is possible that PERK and its downstream PCD regulator, CHOP, do not have functional homologs in Arabidopsis. Besides PERK and CHOP, there is no functional homolog of the BCL-2 family members reported in plants, in particular BAX/BAK, which control mitochondria membrane permeabilization during PCD. There are therefore clear differences in the regulation of ER stressinduced PCD between plant and mammalian cells. However, some ER-stress PCD regulators are conserved between plant cells and mammalian cells such as BAX-inhibitor and it is remarkable that despite the absence of caspase homologs in plants (Watanabe and Lam, 2008) caspase-like activities are induced during ER stress-induced PCD. In particular, caspase-3-like and caspase-9like activities are triggered in soybean suspension cells during ER stress-induced PCD activated using cyclopiazonic acid (CPA), a chemical which inhibits ER Ca ${ }^{2+}$-ATPase and causes $\mathrm{Ca}^{2+}$ efflux from the ER (Zuppini etal., 2004). Also in soybean, overexpression of N-rich proteins NRP-A and NRP-B or of a NAC transcription factor GmNAC81 (former GmNAC6), resulted in the induction of UPR and an increase of caspase-1-like and caspase3-like activity (Costa etal., 2008; Faria et al., 2011). These few reports point out at caspases-like proteases being components of ER stress-induced PCD in plants. The next question is what are the proteases responsible for these caspase-like activities and do they regulate $\mathrm{PCD}$ ?

\section{PLANT CASPASE-LIKE ACTIVITIES AND ER STRESS- INDUCED PCD}

Caspase-like activities has been observed in plant during PCD consistently since their first report (del Pozo and Lam, 1998). However, it took a long period of time to identify plant proteases responsible for the caspase-like activities. Initially, plant scientists pinned their hopes on metacaspases, as metacaspases were identified as distant relatives of animal caspases (Uren et al., 2000). Indeed metacaspases share some structural features with caspases. For example, metacaspases have a catalytic dyad based on cysteine and histidine (Uren etal., 2000). It is now clearly established, however, that metacaspases do not possess caspase-like enzymatic activity at all, as their cleavage site requires an Arg in P1 (R, e.g., RR) rather than Asp (D, e.g., YVAD, DEVD; Vercammen et al., 2004; He etal., 2008; Tsiatsiani et al., 
2011); Other plant proteases were found to exhibit caspase-like activities.

\section{CASPASE-1-LIKE ACTIVITY: VACUOLAR PROCESSING ENZYME}

Vacuolar Processing Enzyme (VPE) is a plant protease localized in the vacuole and responsible for processing vacuolar proteins. Hatsugai et al. (2004) reported that VPE, in addition to its activity against the tetrapeptide ESEN, exhibited caspase-1-like enzymatic activity as shown by activity labeling using the caspase- 1 inhibitor YVAD-CHO. First, protein extracts from Nicotiana benthamiana were labeled with biotin-x-VAD-FMK. Two bands at $40 \mathrm{kDa}$ and $38 \mathrm{kDa}$ were detected and the labeling of these two bands was reduced with the addition of the caspase-1 inhibitor ac-YVAD$\mathrm{CHO}$ or of a VPE antibody (Hatsugai et al., 2004). This indicated that VPE possessed caspase-1-like activity in plants. In addition, the recombinant $\gamma \mathrm{VPE}$ recognized the VPE substrate ESEN with a $\mathrm{Km}$ of $30.3 \mu \mathrm{m}$ and the caspase- 1 substrate YVAD with a $\mathrm{Km}$ of $44.2 \mu \mathrm{m}$ (Kuroyanagi et al., 2005) Further experiments suggested that VPE participate in plant PCD via controlling tonoplast rupture (Hatsugai et al., 2004). The fact that VPE has caspase-1-like activity had been documented for almost ten years, whereas a proof that VPE regulated ER stress induced PCD was only established recently using Piriformospora indica and Arabidopsis as an experimental system. In order for the mutualistic fungi $P$. indica to successfully colonize Arabidopsis roots, roots need to undergo a localized PCD (Qiang et al., 2012). A hallmark of PCD during the colonization of $P$. indica was the collapse of tonoplast. In addition, VPE null mutant reduced PCD as $P$. indica colonized roots (Qiang et al., 2012). Interestingly, Qiang et al. (2012) observed a swelling of ER, which was considered as an indication of ER stress, during $P$. indica colonization. To trigger ER stress-induced PCD, $P$. indica needs to overcome UPR, which tends to dampen the ER stress response. There is evidence that this is done by repressing UPR genes expression during colonization (Qiang et al., 2012). This set of experiments implied that VPE was downstream of UPR and part of the ER-PCD pathway. A recent report brings more details to this model. Two NAC transcription factors in soybean, GmNAC30 and GmNAC81, that induce PCD downstream of osmotic and ER stress, are able to interact with each other in a synergistic manner to activate directly VPE gene expression (Mendes et al., 2013). Both GmNAC30 and GmNAC81 transcript levels increased under ER stress induced using tunicamycin (Mendes et al., 2013).

\section{CASPASE-3-LIKE ACTIVITY: PROTEASOME}

$26 \mathrm{~S}$ proteasome is a proteases complex responsible for protein degradation in eukaryotic cells. The core unit of the $26 \mathrm{~S}$ proteasome is the $20 \mathrm{~S}$ proteasome. It consists of $\alpha$ and $\beta$ subunits. Adding proteasome inhibitors, $\beta$-lactone and APnLD-CHO, to Arabidopsis leaf extract strongly reduced caspase-3-like activity as measured using the substrate DEVD (Hatsugai et al., 2009). In addition, the $20 \mathrm{~S}$ proteasome $\beta$ subunit 1 (PBA1) was detected by antibodies in protein-pull-down using the substrate analog inhibitor biotin-DEVD-FMK (Hatsugai et al., 2009). The activities of proteasome $\beta$ subunit 2 (PBB) and 5 (PBE) were partially repressed by the caspase- 3 inhibitor ac-DEVD-CHO at high concentration (>5 $\mu \mathrm{M}$; Hatsugai et al., 2009). However, the authors suggested that neither PBB nor PBE possesses caspase-3-like activity, because the inhibition of their activities by ac-DEVD-CHO was not dose dependent (Hatsugai et al., 2009). Further to this, Han et al. (2012) purified a caspase-3-like activity from Poplar xylem tissues. In the elution fractions which showed highest caspase3-like activity, the authors detected several proteins and mass spectrum analysis identified those proteins as proteasome subunit homologs in Poplar (Han et al., 2012). In other word, the caspase-3-like activity detected in plants is at least in part due to proteasome activity. In the context of ER stress-induced PCD, one major function of the proteasome is disposing of misfolded proteins in a process termed ER Associated Degradation (ERAD; Liu and Howell, 2010). Blocking proteasome activity is believed to reduce the degradation of misfolded proteins, which in turn causes more burdens to the ER. This implies that PBA1, as a proteasome subunit, may play a pro-survival role rather than a pro-cell death role in plant ER stress-induced PCD despite its caspase-3-like activity. So far, very few reports have depicted the role of the proteasome in PCD. Both pro-cell death and anti-cell death functions have been documented. Silencing PBA1 blocked pathogen-induced PCD in Arabidopsis by blocking one of the first steps consisting of tonoplast and plasma membrane fusion (Hatsugai et al., 2009). On the other hand, silencing the 20 S $\alpha 6$ subunit or the RPN9 subunit of 19 S did elicit PCD in $N$. benthamiana (Kim et al., 2003). Similar contrasting observations have been made in animal cells. Proteasome inhibition using MG132 in human Jurkat cell increased the expression level of BIP, a marker of increased ER stress and resulted in more apoptosis (Park et al., 2011). Another report, based on vascular smooth muscle cells, also indicated that proteasome inhibition sensitized cells to ER stress-induced PCD (Amanso et al., 2011). This may be because applying both an ER stress inducer and a proteasome inhibitor may result not only in more misfolded protein aggregation, but also in a compromised UPR induction. In support of that, cells treated with the proteasome inhibitor MG132 exhibit a repressed UPR genes induction (Lee et al., 2003); how MG132 reduced UPR gene induction is not clear. On the other hand, a pro-apoptotic role of proteasome was also reported. Egger et al. (2007), reported that inhibition of the proteasome using lactacystin block ER stress-induced cell death in Rat 6 embryo fibroblasts (R6; Egger et al., 2007). The authors postulated that proteasome inhibition abolished the degradation of anti-apoptotic BCL-2 family members, so that apoptosis was blocked (Egger et al., 2007). It should be noted that a particular way of inducing ER stress was used in this research, as cells were co-incubated with brefeldin A (BFA) and cycloheximide (CHX). BFA blocks protein transport from ER to Golgi, causing ER stress. CHX represses bulk protein translation including pro-survival UPR proteins, such as proteins downstream of ATF6 (Egger et al., 2007). With this combination of chemicals, protein input into ER was reduced and proteasome inhibition resulted only in a moderate unfolded protein burdens to ER, while BCL-2 degradation was blocked. Our lab is investigating whether the plant proteasome exhibits a pro- or an anti-cell death role in ER stress-induced PCD. Besides the proteasome, our unpublished results indicate that at least one other protease exhibit caspase-3-like activity in Arabidopsis. 
Table 1 | Plant caspase-like activities and ER stress-induced PCD.

\begin{tabular}{|c|c|c|c|c|}
\hline Protease & Preferred caspase substrate & Reference & Species & Comments \\
\hline Vacuolar processing enzyme (VPE) & YVAD, caspase-1-like & $\begin{array}{l}\text { Hatsugai etal. (2004), } \\
\text { Qiang et al. (2012), } \\
\text { Mendes etal. (2013) }\end{array}$ & $\begin{array}{l}\text { Arabidopsis, } \\
\text { soybean, } \\
\text { N. benthamiana }\end{array}$ & $\begin{array}{l}\text { Controls tonoplast rupture } \\
\text { during PCD. Positively } \\
\text { regulate ER stress-induced } \\
\text { PCD. }\end{array}$ \\
\hline Proteasome subunit $\beta$ 1(PBA1) & DEVD, caspase-3-like & $\begin{array}{l}\text { Hatsugai etal. (2009), } \\
\text { Han etal. (2012) }\end{array}$ & Arabidopsis, poplar & $\begin{array}{l}\text { Mediates PCD during HR. } \\
\text { Activity detected during ER } \\
\text { stress-induced PCD }\end{array}$ \\
\hline Phytaspase & VEID, caspase-6-like & Chichkova et al. (2010) & N. tabacum, rice & $\begin{array}{l}\text { Mediates HR and } \\
\text { ROS-induced PCD. Not } \\
\text { reported in ER } \\
\text { stress-induced PCD. }\end{array}$ \\
\hline Saspase & $\begin{array}{l}\text { VEHD, caspase-6-like; IETD, } \\
\text { caspase-8-like }\end{array}$ & $\begin{array}{l}\text { Coffeen and Wolpert } \\
\text { (2004) }\end{array}$ & Oat & $\begin{array}{l}\text { Not reported in ER } \\
\text { stress-induced PCD }\end{array}$ \\
\hline
\end{tabular}

\section{OTHER CASPASE-LIKE ACTIVITIES}

Besides the proteases responsible for caspase-1 and caspase-3-like activities involved in ER stress induced PCD, other proteases with caspase-like activities are known in plants. Phytaspase and saspase were found to exhibit caspase-like activities (Vartapetian et al., 2011). So far, however, no report has shown the involvement of these proteases in ER stress-induced PCD. Phytaspase, a subtilisin-like serine protease, takes its name from being a plant ASPartate-specific protease and was purified from tobacco and rice. It exhibits a preferential cleavage activity against caspase6 substrate VEID although it can cleave other caspase substrates such as caspase 1 (YVAD) and 9 (LEHD) in particular (Chichkova et al., 2010). Phytaspase was shown to mediate HR-induced PCD and ROS-induced PCD (Chichkova et al., 2010). Saspase (Serine protease with an asp cleavage site) is a subtilisin-like serine protease, purified from Avena sativa, that exhibits an enzymatic activity against caspase- 6 substrates (VEHD, VKMD, VNLD) and one caspase-8 substrate (IETD; Coffeen and Wolpert, 2004). A role for caspase- 2 and caspase- 4 have been described in mammalian ER stress, but caspase-2-like or caspase-4-like activity has not been reported in plants. As caspase-2 was reported to regulate ER stress-induced apoptosis in mammalian cells (Upton et al., 2012), caspase-2 activity could be tested in plant during ER stress-induced PCD although this activity in itself does not indicate a role in plant PCD. The caspase-like activities in plants and their correlations with ER stress-induced PCD are summarized in Table 1.

\section{CONCLUSION}

ER stress-induced PCD is gaining more and more attention in plant science research. So far, among plant proteases with caspase-like activity, only VPE, with a caspase-1-like activity, has been implicated in regulating ER stress-induced PCD. VPE is proposed to control indirectly tonoplast rupture during $\mathrm{PCD}$. The detailed mechanism by which VPE controls tonoplast rupture is still unclear and could be addressed using ER-stress induced PCD as an experimental system. Another aspect that can be addressed is the role of caspase-3-like activity and of the proteasome. In particular, it is worth investigating whether in addition to being responsible for the degradation of misfolded proteins, the proteasome could also indirectly modulate UPR gene expression as suggested in some mammalian cell models (Lee et al., 2003; Amanso et al., 2011). Since three caspase-like activities have been directly or indirectly implicated in ER stress-induced PCD in plants, a complete profile of caspase-like activities during ER stress-induced PCD could be carried out using substrates and inhibitors. Following this, using relevant $\mathrm{KO}$ lines or KD lines would provide clearer experimental outcomes. Such an approach would give an insight into which additional proteases are involved in ER stress-induced PCD in plants. For example, whether phytaspase, a PCD regulator, has a function in ER stress-induced PCD is an interesting question that is still open. Finally, a complete understanding will not be achieved without knowledge of the in vivo protein substrates implicated.

\section{ACKNOWLEDGMENT}

Our research is funded by the BBSRC.

\section{REFERENCES}

Amanso, A. M., Debbas, V., and Laurindo, F. R. M. (2011). Proteasome inhibition represses unfolded protein response and Nox4, sensitizing vascular cells to endoplasmic reticulum stress-induced death. PLoS ONE 6:e14591. doi: 10.1371/journal.pone.0014591

Bollhöner, B., Prestele, J., and Tuominen, H. (2012). Xylem cell death: emerging understanding of regulation and function. J. Exp. Bot. 63, 1081-1094. doi: $10.1093 /$ jxb/err438

Chichkova, N. V., Shaw, J., Galiullina, R. A., Drury, G. E., Tuzhikov, A. I., Kim, S. H., et al. (2010). Phytaspase, a relocalisable cell death promoting plant protease with caspase specificity. EMBO J. 29, 1149-1161. doi: 10.1038/emboj.2010.1

Coffeen, W. C., and Wolpert, T. J. (2004). Purification and characterization of serine sroteases that exhibit caspase-like activity and are associated with programmed cell Death in Avena sativa. Plant Cell 16, 857-873. doi: 10.1105/tpc. 017947

Costa, M. D. L., Reis, P. A. B., Valente, M. A. S., Irsigler, A. S. T., Carvalho, C. M., Loureiro, M. E., et al. (2008). A new branch of endoplasmic reticulum 
stress signaling and the osmotic signal converge on plant-specific asparaginerich proteins to promote cell death. J. Biol. Chem. 283, 20209-20219. doi: 10.1074/jbc.M802654200

Davis, R. J. (2000). Signal transduction by the JNK group of MAP kinases. Cell 103 239-252. doi: 10.1016/S0092-8674(00)00116-1

del Pozo, O., and Lam, E. (1998). Caspases and programmed cell death in the hypersensitive response of plants to pathogens. Curr. Biol. 8, 1129-1132. doi: 10.1016/S0960-9822(98)70469-5

Duan, Y., Zhang, W., Li, B., Wang, Y., Li, K., Sodmergen, Han, C., et al. (2010). An endoplasmic reticulum response pathway mediates programmed cell death of root tip induced by water stress in Arabidopsis. New Phytol. 186, 681-695. doi: 10.1111/j.1469-8137.2010.03207.x

Egger, L., Madden, D. T., Rhême, C., Rao, R. V., and Bredesen, D. E. (2007) Endoplasmic reticulum stress-induced cell death mediated by the proteasome. Cell Death Differ. 14, 1172-1180. doi: 10.1038/sj.cdd.4402125

Faria, J., Reis, P., Reis, M. T., Rosado, G., Pinheiro, G., Mendes, G., et al. (2011). The NAC domain-containing protein, GmNAC6, is a downstream component of the ER stress- and osmotic stress-induced NRP-mediated cell-death signaling pathway. BMC Plant Biol. 11:129. doi: 10.1186/1471-2229-11-129

Greenberg, J. T. (1996). Programmed cell death: a way of life for plants. Proc. Natl. Acad. Sci. 93, 12094-12097. doi: 10.1073/pnas.93.22.12094

Groover, A., DeWitt, N., Heidel, A., and Jones, A. (1997). Programmed cell death of plant tracheary elements differentiating in vitro. Protoplasma 196, 197-211. doi: 10.1007/BF01279568

Han, J.-J., Lin, W., Oda, Y., Cui, K. -M., Fukuda, H., and He, X. -Q. (2012). The proteasome is responsible for caspase-3-like activity during xylem development. Plant J. 72, 129-141. doi: 10.1111/j.1365-313X.2012.05070.x

Harding, H. P., Zhang, Y., and Ron, D. (1999). Protein translation and folding are coupled by an endoplasmic-reticulum-resident kinase. Nature 397, 271-274. doi: $10.1038 / 16729$

Harding, H. P., Zhang, Y., Bertolotti, A., Zeng, H., and Ron, D. (2000). Perk is essential for translational regulation and cell survival during the unfolded protein response. Mol. Cell 5, 897-904. doi: 10.1016/S1097-2765(00)80330-5

Harding, H. P., Zhang, Y., Zeng, H., Novoa, I., Lu, P. D., Calfon, M., et al. (2003). An integrated stress response regulates amino acid metabolism and resistance to oxidative stress. Mol. Cell 11, 619-633. doi: 10.1016/S1097-2765(03)00105-9

Hatsugai, N., Kuroyanagi, M., Yamada, K., Meshi, T., Tsuda, S., Kondo, M., et al. (2004). A plant vacuolar protease, vpe, mediates virus-induced hypersensitive cell death. Science 305, 855-858. doi: 10.1126/science.1099859

Hatsugai, N., Iwasaki, S., Tamura, K., Kondo, M., Fuji, K., Ogasawara, K., et al. (2009). A novel membrane fusion-mediated plant immunity against bacterial pathogens. Genes Dev. 23, 2496-2506. doi: 10.1101/gad.1825209

He, R., Drury, G. E., Rotari, V. I., Gordon, A., Willer, M., Farzaneh, T., et al. (2008). Metacaspase- 8 modulates programmed cell death induced by ultraviolet light and H2O2 in Arabidopsis. J. Biol. Chem. 283, 774-783. doi: 10.1074/jbc.M704185200

Howell, S. H. (2013). Endoplasmic Reticulum Stress Responses in Plants. Annu. Rev. Plant Biol. 64, 477-499. doi: 10.1146/annurev-arplant-050312-120053

Kamauchi, S., Nakatani, H., Nakano, C., and Urade, R. (2005). Gene expression in response to endoplasmic reticulum stress in Arabidopsis thaliana. FEBS J. 272, 3461-3476. doi: 10.1111/j.1742-4658.2005.04770.x

Kim, M., Ahn, J.-W., Jin, U.-H., Choi, D., Paek, K.-H., and Pai, H.-S. (2003). Activation of the programmed cell death pathway by inhibition of proteasome function in plants. J. Biol. Chem. 278, 19406-19415. doi: 10.1074/jbc.M210539200

Kuroyanagi, M., Yamada, K., Hatsugai, N., Kondo, M., Nishimura, M., and HaraNishimura, I. (2005). Vacuolar processing enzyme is essential for mycotoxininduced cell death in Arabidopsis thaliana. J. Biol. Chem. 280, 32914-32920. doi: 10.1074/jbc.M504476200

Lee, A.-H., Iwakoshi, N. N., Anderson, K. C., and Glimcher, L. H. (2003). Proteasome inhibitors disrupt the unfolded protein response in myeloma cells. Proc. Natl. Acad. Sci. 100, 9946-9951. doi: 10.1073/pnas.1334037100

Liu, J.-X., and Howell, S. H. (2010). Endoplasmic reticulum protein quality control and its relationship to environmental stress responses in plants. Plant Cell Online 22, 2930-2942. doi: 10.1105/tpc.110.078154

McStay, G. P., Salvesen, G. S., and Green, D. R. (2007). Overlapping cleavage motif selectivity of caspases: implications for analysis of apoptotic pathways. Cell Death Differ. 15, 322-331.doi: 10.1038/sj.cdd.4402260

Mendes, G. C., Reis, P. A. B., Calil, I. P., Carvalho, H. H., Aragão, F. J. L., and Fontes, E. P. B. (2013). GmNAC30 and GmNAC81 integrate the endoplasmic reticulum stress- and osmotic stress-induced cell death responses through a vacuolar processing enzyme. Proc. Natl. Acad. Sci. U.S.A. 110, 19627-19632. doi: 10.1073/pnas.1311729110

Moreno, A. A., Mukhtar, M. S., Blanco, F., Boatwright, J. L., Moreno, I., Jordan, M. R., et al. (2012). IRE1/bZIP60-mediated unfolded protein response plays distinct roles in plant immunity and abiotic stress responses. PLoS ONE 7:e31944. doi: 10.1371/journal.pone.0031944

Nakagawa, T., Zhu, H., Morishima, N., Li, E., Xu, J., Yankner, B. A., et al. (2000) Caspase-12 mediates endoplasmic-reticulum-specific apoptosis and cytotoxicity by amyloid- $\beta$. Nature 403, 98-103. doi: 10.1038/47513

Nishitoh, H., Saitoh, M., Mochida, Y., Takeda, K., Nakano, H., Rothe, M., et al. (1998). ASK1 is essential for JNK/SAPK activation by TRAF2. Mol. Cell 2, 389395. doi: 10.1016/S1097-2765(00)80283-X

Park, H. S., Jun, D. Y., Han, C. R., Woo, H. J., and Kim, Y. H. (2011). Proteasome inhibitor MG132-induced apoptosis via ER stress-mediated apoptotic pathway and its potentiation by protein tyrosine kinase p56lck in human Jurkat T cells. Biochem. Pharmacol. 82, 1110-1125. doi: 10.1016/j.bcp.2011.07.085

Qiang, X., Zechmann, B., Reitz, M. U., Kogel, K.-H., and Schäfer, P. (2012). The mutualistic fungus piriformospora indica colonizes Arabidopsis roots by inducing an endoplasmic reticulum stress triggered caspase-dependent cell death. Plant Cell 24, 794-809. doi: 10.1105/tpc.111.093260

Rotari, V. I., Dando, P. M., and Barrett, A. J. (2001). Legumain forms from plants and animals differ in their specificity. Biol. Chem. 382, 953-959. doi: 10.1515/BC.2001.119

Szegezdi, E., Logue, S. E., Gorman, A. M., and Samali, A. (2006). Mediators of endoplasmic reticulum stress-induced apoptosis. EMBO Rep. 7, 880-885. doi: 10.1038/sj.embor.7400779

Tsiatsiani, L., Van Breusegem, F., Gallois, P., Zavialov, A., Lam, E., and Bozhkov, P. V. (2011). Metacaspases. Cell Death Differ. 18, 1279-1288. doi: 10.1038/cdd. 2011.66

Upton, J.-P., Wang, L., Han, D., Wang, E. S., Huskey, N. E., Lim, L., et al. (2012). IRE1 $\alpha$ cleaves select microRNAs during ER stress to derepress translation of proapoptotic caspase-2. Science 338, 818-822. doi: 10.1126/science.12 26191

Uren, A. G., O’Rourke, K., Aravind, L., Pisabarro, M. T., Seshagiri, S., Koonin, E. V., et al. (2000). Identification of paracaspases and metacaspases: two ancient families of caspase-like proteins, one of which plays a key role in malt lymphoma. Mol. Cell 6, 961-967.

Van der Hoorn, R. A. L. (2008). Plant proteases: from phenotypes to molecular mechanisms. Annu. Rev. Plant Biol. 59, 191-223. doi: 10.1146/annurev.arplant.59.032607.092835

Vartapetian, A. B., Tuzhikov, A. I., Chichkova, N. V., Taliansky, M., and Wolpert, T. J. (2011). A plant alternative to animal caspases: subtilisin-like proteases. Cell Death Differ. 18, 1289-1297. doi: 10.1038/cdd.2011.49

Vercammen, D., van de Cotte, B., De Jaeger, G., Eeckhout, D., Casteels, P., Vandepoele, K., et al. (2004). Type II metacaspases Atmc4 and Atmc9 of Arabidopsis thaliana cleave substrates after arginine and lysine. J. Biol. Chem. 279, 45329-45336. doi: 10.1074/jbc.M406329200

Watanabe, N., and Lam, E. (2008). BAX inhibitor-1 modulates endoplasmic reticulum stress-mediated programmed cell death in Arabidopsis. J. Biol. Chem. 283, 3200-3210. doi: 10.1074/jbc.M706659200

Xu, Q., and Zhang, L. (2009). Plant caspase-like proteases in plant programmed cell death. Plant Signal. Behav. 4, 902-904. doi: 10.4161/psb.4.9.9531

Yoshida, H., Okada, T., Haze, K., Yanagi, H., Yura, T., Negishi, M., et al. (2000). ATF6 activated by proteolysis binds in the presence of NF-Y (CBF) directly to the cis-acting element responsible for the mammalian unfolded protein response. Mol. Cell. Biol. 20, 6755-6767. doi: 10.1128/MCB.20.18.6755-676 7.2000

Zinszner, H., Kuroda, M., Wang, X., Batchvarova, N., Lightfoot, R. T., Remotti, H., et al. (1998). CHOP is implicated in programmed cell death in response to impaired function of the endoplasmic reticulum. Genes Dev. 12, 982-995. doi: 10.1101/gad.12.7.982

Zuppini, A., Navazio, L., and Mariani, P. (2004). Endoplasmic reticulum stressinduced programmed cell death in soybean cells. J. Cell Sci. 117, 2591-2598. doi: $10.1242 /$ jcs. 01126

Conflict of Interest Statement: The authors declare that the research was conducted in the absence of any commercial or financial relationships that could be construed as a potential conflict of interest. 
Received: 04 December 2013; accepted: 28 January 2014; published online: 14 February 2014.

Citation: Cai Y-M, Yu J and Gallois P (2014) Endoplasmic reticulum stress-induced PCD and caspase-like activities involved. Front. Plant Sci. 5:41. doi: 10.3389/fpls.2014. 00041

This article was submitted to Plant Cell Biology, a section of the journal Frontiers in Plant Science.
Copyright (c) 2014 Cai, Yu and Gallois. This is an open-access article distributed under the terms of the Creative Commons Attribution License (CC BY). The use, distribution or reproduction in other forums is permitted, provided the original author(s) or licensor are credited and that the original publication in this journal is cited, in accordance with accepted academic practice. No use, distribution or reproduction is permitted which does not comply with these terms. 